\title{
LES VARIETES DU FRANÇAIS EN LOUISIANE ET LEUR STATUT SOCIOLINGUISTIQUE
}

\author{
Chimegsaikhan Banzar \\ Grambling State University \\ banzarc@gram.edu
}

\begin{abstract}
Le français en Louisiane est parlé depuis la colonisation de ce territoire par la France, à partir du XVII ${ }^{\mathrm{e}} \mathrm{s}$. Au cours de son histoire, la Louisiane a connu au moins quatre variétés du français: français colonial ${ }^{1}$, français cadien, français créole louisianais et français standard, où les trois premières sont des variétés vernaculaires. En raison de contacts linguistiques des populations, de leurs interférence et assimilation l'une à l'autre, ainsi que de la forte présence de variations intracommunautaires, aujourd'hui il est difficile de tracer une nette ligne de démarcation entre ces vernaculaires. Néanmoins, il existe une hiérarchisation sociolinguistique et socioethnique importante qui caractérise les variétés franco-louisianaises. La communication a pour objectif de démontrer l'usage différentiel de ces parlers en fonction de leurs statuts sociolinguistiques respectifs. Au départ, l'auteur fait une brève analyse des origines et de l'évolution de chacune des variétés du français, puis une étude sociologique de leur utilisation, et en conclusion, elle discutera de la stratification sociolinguistique de ces parlers où le français standard occupe une place privilégiée face aux autres trois variétés qui constituent le patrimoine linguistique de la Louisiane francophone.
\end{abstract}

\section{Le français colonial et le français standard}

Le français colonial ${ }^{2}$ était parlé par les premiers colons, installés à la Nouvelle-Orléans et les régions fluviales du bas du Mississipi, et leurs descendants, appelés Créoles. C'étaient principalement des planteurs venus de France ou exilés de Saint Domingue qui, à l'époque coloniale, possédaient des terres et des esclaves. Représentant l'aristocratie ayant fui la Révolution française (dont font preuve les noms de famille comme de la Houssaye, de Chastaigne, de la Loire, du Quesnay, de Clouet), ces vieilles familles de souche française constituaient la haute société louisianaise éduquée, qui parlait un français soutenu. Cette variété de français parlée par les élites blanches reproduisait le rapport de forces dans une société coloniale. Le système économique de plantations était basé sur une structure sociale très hiérarchisée: la classe dominante était représentée par les planteurs qui possédaient des terres et des esclaves, et la classe dominée, par les esclaves, qui travaillaient dans les champs pour leurs maîtres. Entre ces deux classes principales, il existait une strate moyenne, composée d'artisans (forgerons, charpentiers) et de métayers (Haas 25). Dans cette hiérarchie sociale, les planteurs créoles occupaient évidemment la position supérieure, donc la variété qu'ils parlaient, le français colonial, était prestigieuse et la plus valorisée (Figure 1). C'était la langue de haute culture dans laquelle les Créoles composaient leurs œuvres littéraires et artistiques et publiaient les revues et les journaux comme L'Abeille, le Courrier de la Nouvelle-Orléans et bien d'autres. De ce fait, le français qu'ils parlaient, écrivaient et lisaient au début était un français indistinct de celui de France. Les œuvres d'Alfred Mercier, de Félix Voorhies, de Madame de la Houssaye, de Louise Fortier constituent le patrimoine littéraire francolouisianais de cette élite. Par sa forme écrite, le français colonial ne se distinguait pas du français standard comme le témoignent les écrits louisianais du XIX ${ }^{\mathrm{e}}$ siècle. Cependant, à l'oral, le français colonial se distinguait par un certain accent et une "légère intonation musicale" (Lauvrière 431). Smith-Thibodeaux note « [le] rythme plus lent et [les] voyelles (e, o, notamment) plus longues que dans le français de France » (48). Quant au vocabulaire, il distingue «trois éléments principaux: une anglicisation relativement faible ("Il me 
tarde de ..." employé pour "je suis en retard pour ...”) [...]; un recours à des mots anciens; une créativité lexicale très française, et l'attribution de nouvelles significations à des mots déjà existants mais tout à fait dans "l'esprit de la langue": une "banquette" est un trottoir, un "îlet" est un pâté de maisons - ce qui traduit le terme anglais block» (48).

De nos jours, cette variété a presque disparu et n'est parlée que par la vieille génération de Créoles de la Nouvelle-Orléans, de Donaldsville, de Saint Martinville ou dans certaines localités rurales, comme le village la Vacherie (Paroisse de Saint-James). Gérard Saint-Martin, ancien professeur de français à l'Université de la Louisiane du Sud-Ouest (aujourd'hui l'Université de la Louisiane à Lafayette), constatait la disparition de ce parler déjà en 1983 (101). A l'opposé des autres variétés vernaculaires de la Louisiane, la vitalité du français colonial a été précaire: sous l'influence de l'américanisation, les élites créoles ont été les premières à adopter la langue et la culture dominantes.

Aujourd'hui, dans le cadre de la revitalisation du français en Louisiane débutée en 1968, la variété qui est privilégiée dans le système scolaire de l'Etat est le français standard tel qu'il est parlé dans l'Hexagone. Le choix du français standard par les autorités louisianaises comme véhicule de la revitalisation de la langue française en Louisiane a été dicté par des intérêts pratiques, "au profit culturel, économique et touristique de 1'Etat" (Acte législatif 409, State of Louisiana, 1968). Le français standard est devenu un atout important pour les Louisianais, une condition-clé de l'enrichissement culturel et économique.

\section{Le français cadien}

Le français cadien est une variété vernaculaire parlée par la population francophone de l'Acadiana, région du sud-ouest de la Louisiane. Originaires de l'Acadie, les Cadiens se sont installés en Louisiane au XVIIIe siècle et ont su conserver leur langue grâce à l'isolement géographique, au mode de vie rural, aux fortes traditions familiales et religieuses. En contact avec d'autres groupes ethniques, les immigrés européens, les Amérindiens et les Créoles de couleur, les Cadiens ont par ailleurs été fortement influencés par ces diverses langues et cultures, notamment dans le domaine lexical qui a été enrichi par des emprunts à l'espagnol (bacalao, lagniappe, chaurice), aux langues amérindiennes (bayou, ouaouaron, chaoui) et africaines (gombo, gri-gri, voudou). Inversement, par son influence substantielle sur les autres minorités ethniques (les Allemands, Irlandais, Espagnols et Amérindiens), le français cadien est devenu une langue véhiculaire non seulement pour les Cadiens mais aussi pour de nombreuses communautés environnantes des bayous et des prairies de la Louisiane du sud. Selon le recensement fédéral de 2000, la population de la Louisiane comptait 4153367 habitants dont 194 mille soit 4,7\% parlaient le français à la maison, y compris le cadien ${ }^{3}$.

Le français cadien n'a pas évité les deux grands courants linguistiques des derniers siècles dont parle Labov: "le déclin des dialectes locaux et l'accroissement de la stratification verticale de la langue" (Sociolinguistique 402). La première tendance se manifeste par le déclin du français cadien provoqué d'une part, par l'assimilation linguistique des Cadiens à l'anglais, langue dominante, et d'autre part, par la marginalisation des variétés dialectales à l'intérieur de la même langue (français standard vs variétés vernaculaires). Avec l'implantation de l'anglais dans toutes les sphères de la société louisianaise et l'anglicisation de l'instruction publique et non-publique, les fonctions sociales du français cadien ont été réduites à un usage très restreint. D'autre part, la survalorisation du français standard comme un modèle "correct, prestigieux, moderne, profitable, rentable etc." a porté préjudice à la vitalité des langues vernaculaires. La deuxième tendance est liée à la hiérarchisation sociolinguistique des variétés franco-louisianaises que nous examinons plus loin.

Les jugements dévalorisants du français cadien comme d'un français "rural”, "vieilli", "archaïque", d'un français du XVII ${ }^{\mathrm{e}}-\mathrm{XVIII}$ ' siècles, d'un "mauvais français", ont contribué activement à l'insécurité linguistique 
de cette communauté. Cette dévalorisation s'explique par une série de facteurs sociolinguistiques. Premièrement, par son statut de langue vernaculaire, qui se distingue du français standard par des attributs linguistiques singuliers au niveau du lexique, de la phonologie, des structures grammaticales. Deuxièmement, par le fait que le français cadien est une langue orale. Troisièmement, par le haut niveau d'analphabétisme des Cadiens au XVIIIe-XIXe siècles et leur manque d'éducation formelle. Et, enfin, par le statut social inférieur des Cadiens, en majorité agriculteurs, pêcheurs, chasseurs, trappeurs, petits artisans, par rapport aux planteurs créoles. Ainsi, par association et extension, la langue des Cadiens s'est retrouvée doublement infériorisée: par rapport à la langue de l'élite francophone et par rapport à l'anglais.

Sur le plan sociolinguistique, le français cadien garde toujours le statut de langue vernaculaire, d'un parler propre à une communauté locale. De même que dans le passé, il se retrouve aujourd'hui doublement marginalisé: face à l'anglais, langue dominante, et au français standard, officiellement proclamé langue seconde de la Louisiane en 1968.

Progressivement évincé par les langues dominantes au pouvoir politique et socio-économique, le français cadien est parlé aujourd'hui en famille, principalement en milieu rural, parmi les chasseurs, pêcheurs, trappeurs des paroisses francophones. Cependant, avec la renaissance de la culture et de la langue cadiennes, un renouveau d'intérêt pour leur patrimoine linguistique est attesté chez les jeunes Cadiens qui ont de plus en plus tendance à parler le français cadien dans les endroits publics comme cafés, restaurants, magasins ou cinémas. Ce changement dans les pratiques linguistiques des jeunes a été signalé par plusieurs linguistes. Les recherches sociolinguistiques de Condon et de Pittman (Language Attitudes) révèlent une attitude plus favorable envers le français cadien parmi les jeunes de moins de 21 ans que parmi les groupes plus âgés (2139 ans et 40-60 ans). Les enquêteurs lient ce phénomène aux effets de la popularisation de la culture cadienne comme musique, danse et cuisine des Cajuns (Blyth 33). Ces manifestations d'un renouveau d'intérêt pour le français cadien permettent de parler de sa future réhabilitation.

\section{Le français créole louisianais}

Le français créole louisianais est une variété vernaculaire majoritairement parlée par les descendants d'esclaves africains qui ont été amenés en Louisiane au XVIII $-\mathrm{XIX}^{\mathrm{e}}$ siècles. Le créole était une langue de plantations créée par les esclaves au contact avec le français de leurs maîtres. Cette langue est basée sur un vocabulaire à base française, combiné avec des mots africains, antillais et amérindiens. La structure grammaticale pourrait avoir un substrat africain (mais cette question est encore controversée), avec des éléments français simplifiés (Hall, Tisch 44). Becky Brown note qu'à la différence du français colonial et du français cadien qui relèvent d'une hybridation primaire, le français créole louisianais représente un exemple d'une hybridation secondaire (The Development 226).

Le français créole louisianais est donc un parler à dérivation secondaire, basé sur le français colonial qui dérive, à son tour, du français de France. Cette particularité étymologique explique en partie le fait que, lexicalement et grammaticalement, le français créole louisianais est plus distancé de ses origines françaises que les deux autres variétés vernaculaires. De l'autre côté, pour les langues africaines, le créole présente un modèle d'une dérivation primaire. Bien que les esclaves de la Louisiane aient été importés de l'ouest de l'Afrique (Sénégal, Congo), Marshall note une ressemblance significative entre le français créole de la Louisiane et le créole de la Réunion (Océan Indien) (The Origin 338-339). Cette remarque renforce l'idée sur la multiplicité de langues africaines ayant participé à la formation du créole louisianais, ce qui aurait conditionné l'existence de plusieurs variations à l'intérieur du même vernaculaire. Ce continuum de variations s'explique par l'influence des autres variétés vernaculaires franco-louisianaises (français colonial et français 
cadien), sur le créole louisianais, ainsi que par des contextes sociaux différents dans lesquels ces variations ont émergé (Marshall, The Origin 339).

A l'époque coloniale, le créole servait de "lingua franca" non seulement entre les maîtres et leurs esclaves mais aussi entre les esclaves de différentes plantations qui maintenaient des contacts socioculturels et commerciaux étroits (Marshall, The Origin 340). Le français créole louisianais était parlé également par les petits Créoles blancs élevés par les servantes de couleur. Les domestiques, de leur part aussi, apprenaient la langue du maître, le français colonial, et étaient capables de converser en deux langues (variétés), en passant d'une langue à l'autre sans difficulté. Les maîtres également, pour être mieux compris, recouraient au parler des esclaves. Par ailleurs, la société de plantation était une société hiérarchisée composée, à part les maîtres et leurs esclaves, de Blancs pauvres labourant côte à côte avec les esclaves. Ceci explique le fait qu'aujourd'hui le créole louisianais est parlé par les Francophones blancs dans quatre régions: les bas du Mississipi, sur la Côte des Allemands située entre la Nouvelle-Orléans et Bâton Rouge, à Lacombe de la Paroisse de Saint Tammany, dans la Paroisse de Pointe Coupée et dans la région de Bayou Teche de la Paroisse de Saint Martin (Marshall, The Origin 344).

La hiérarchie sociale crée la hiérarchie linguistique: du fait qu'il est parlé par les couches sociales les plus défavorisées, le français créole louisianais a le statut sociolinguistique le plus bas. Il est plus stigmatisé que le français cadien à cause de son association avec les gens de couleur et l'esclavage. Les Créolophones blancs préfèrent le cadien au français créole louisianais (Haas 28). Haas remarque aussi que dans certaines régions comme Bayou Bœuf, la population parle les deux variétés comme "deux formes de la même langue" qui s'emploient alternativement en fonction des divisions géographiques et sociales (29). Selon Tentchoff, les Noirs et les Blancs francophones ont une définition du français créole louisianais beaucoup plus "sociale et raciale" que linguistique. La coexistence des deux parlers relève de leur association à deux systèmes de valeurs. Le français cadien serait associé à la respectabilité et au genre féminin. Le français créole louisianais serait considéré comme une langue masculine et non respectable. Ce dernier serait la langue de la forêt et des marais ainsi que la langue des bars et des exploits masculins (100).

Toutes les deux variétés sont parlées par les classes inférieures de la société louisianaise, mais le français créole louisianais porte des marques plutôt socioethniques que sociolinguistiques. De ce fait, le français créole louisianais, parlé par les esclaves, gens de couleur, est situé en toute dernière position dans la hiérarchie sociale et linguistique:

$\underline{\text { Stratification socioethnique }}$

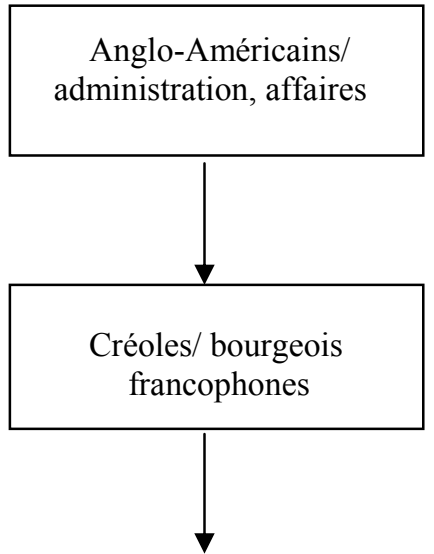

Hiérarchisation dans les représentations linguistiques

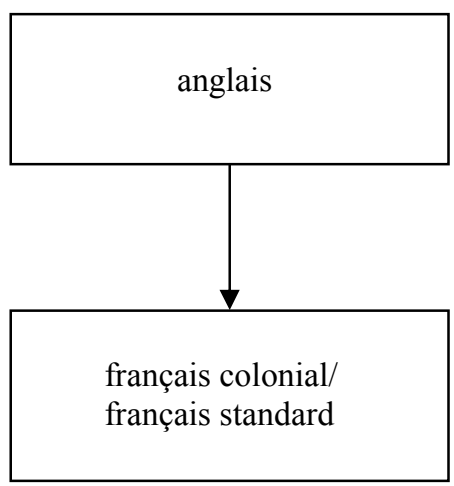



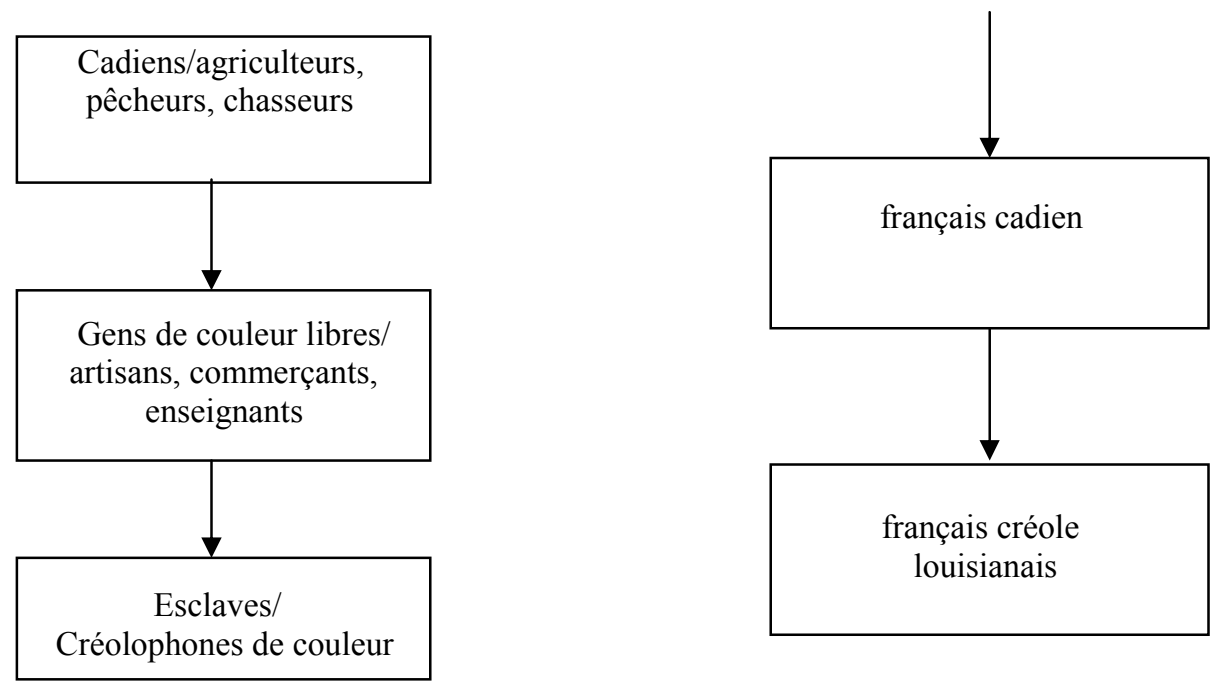

Figure 1: Stratification socioethnique et hiérarchisation dans les représentations linguistiques de la population louisianaise au XVIIIe-XIXe siècles

Au cours de plus de deux siècles de contact avec les langues environnantes, le créole louisianais a intégré des éléments lexico-grammaticaux du français cadien, de l'anglais, des langues amérindiennes, ce qui se manifeste par l'abondance d'emprunts et l'usage fréquent de calques et de l'alternance codique. Cette interférence a résulté en un brouillage progressif des frontières linguistiques entre les deux variétés vernaculaires, le français créole louisianais et le français cadien (Valdman and Klingler 111).

Cependant, les frontières sociolinguistiques et ethniques persistent encore de nos jours. Aujourd'hui, le créole n'est parlé qu'en famille, en petite communauté. Parmi les Louisianais de couleur, la majorité, soit $85 \%$, sont anglophones et seulement 7,5\% francophones (Census of Population 1990). Selon le dernier recensement (2000), la Louisiane comptait 4470 Créolophones. Le déclin du nombre de Créolophones en Louisiane présente un phénomène commun qui caractérise le français cadien et les langues minoritaires en général.

\section{Le statut sociolinguistique des variétés franco-louisianaises}

La présence de trois variétés vernaculaires du français en Louisiane (français colonial, français cadien et français créole louisianais) permet de parler d'un continuum de variations réunies sous le terme de français louisianais. Valdman explique:

Dans la Louisiane dite francophone on distingue généralement trois variétés vernaculaires liées au français: le créole, le cadien et le français colonial. [...] De plus en plus, les spécialistes se servent du terme de français louisianais pour recouvrir toutes les variétés vernaculaires de français. En ce qui concerne la distinction entre ce français louisianais et le créole, la connaissance de ces deux parlers chez les mêmes personnes et les alternances codiques qui en résultent rendent difficile une démarcation nette. Le FL recouvre un continuum de variation dont les deux pôles opposés sont formés par le français standard et le créole et le centre constitué par le cadien. (Le rôle 154) 
Du point de vue sociolinguistique, ce continuum représenterait une stratification verticale où le français standard occupe le sommet, le français cadien se retrouve au milieu, et le français créole louisianais en bas de l'échelle sociale (Figure 1). Les frontières entre les parlers vernaculaires dépendent aussi de considérations idéologiques: si le français colonial était la langue de l'élite, le français créole louisianais est toujours associé à une langue de la population de couleur à statut socio-économique inférieur. La distinction linguistique reflétait la distinction sociale et raciale et reproduisait les rapports sociaux de race.

Brown propose une autre approche, une approche multidimensionnelle dans l'étude des rapports entre différentes variétés du français louisianais, y compris le cadien créolisé et le créole cadiénisé:

La Francophonie en Louisiane comprend le français international, le cadien et le créole (en proportions différentes). Par conséquent, une tension constante s'exerce entre l'anglais et les variétés françaises. Le résultat n'est pas un continuum linéaire allant du créole au français international mais un autre type de continuum (ou de continua simultanément applicable) dans un espace multidimensionnel tel qu'au Belize, où les locuteurs reconnaissent le code ou la variante approprié(e) selon le contexte social (Les conséquences 54).

Bien que chacune des variétés franco-louisianaises ait des fonctions sociales spécifiques (le français standard comme langue internationale dans les affaires officielles et administratives, dans l'enseignement et le tourisme; le français cadien comme langue du foyer réservée principalement aux Francophones blancs, et le français créole louisianais comme parler de la population de couleur), la question de l'intelligibilité dépend considérablement des attitudes envers la variété donnée.

Les rapports sociaux inégaux caractérisent non seulement les rapports entre diverses variétés de la même langue, mais aussi entre deux systèmes linguistiques distincts, le français et l'anglais. Ce sujet pourrait servir d'objet d'une autre étude sociolinguistique sur le français de la Louisiane.

\section{Références bibliographiques}

Brown, Becky. (1996). Les conséquences théoriques d'un continuum linguistique en Louisiane française. Plurilinguismes, $11,37-62$.

Brown, Becky. (1997). The Development of a Louisiana French Norm. In Valdman, Albert (Ed.). French and Creole In Louisiana, New York: Plenum Press, 226.

Condon, S. and P. Pittman. (1992). Language Attitudes in Southern Louisiana. Ms. Lafayette: U of Southwestern Louisiana.

Haas, David F. (1980). La langue française dans un village du couloir industriel du Mississippi. Document de travail/Working Paper. Projet Louisiane, 9, 22-36.

Hall, G. M. (1992). Africans in Colonial Louisiana: The Development of Afro-Creole Culture in the Eighteenth Century. Baton Rouge: LSU Press.

Labov, William. (1976). Sociolinguistique. Paris: Minuit.

Marshall, Margaret M. (1996). Le rôle de la langue française en Louisiane de ses origines jusqu'aux temps présents. Plurilinguismes, 11, 7-35.

Marshall, Margaret M. (1997). The Origin and Development of Louisiana Creole French. In Valdman, Albert (Ed.). French and Creole in Louisiana, New York: Plenum Press, 338-344.

Smith-Thibodeaux, John. (1977). Les Francophones de Louisiane. Paris : Ed. Entente.

State of Louisiana. (1968). Acts of the Legislature: Regular Session 1968. 3 vol. Baton Rouge: By Authority. 
Tentchoff, Dorice. (1977). Speech in a Louisiana Cajun Community. Diss. Case Western Reserve U.

Tisch, Joseph LeSage. (1959). French in Louisiana. New Orleans: A. F. Laborde and Sons.

Valdman, Albert. (1996). Le rôle de l'enseignement dans la survie du français en Louisiane. Plurilinguismes, 11, 153-177.

Valdman, Albert, and Thomas A. Klingler. (1997). "The Structure of Louisiana Creole." In Valdman, Albert (Ed.). French and Creole in Louisiana, New York: Plenum Press, 111.

${ }^{1}$ La question de la classification du français colonial comme variété spécifique de français est controversée. En raison de sa similitude avec le français standard, le français colonial n'est pas reconnu comme tel par certains linguistes.

${ }^{2}$ Dans les études sur la situation linguistique de la Louisiane, cette variété est appelée de deux manières, soit le français colonial (Valdman, Le rôle de l'enseignement 154), soit le français créole (Marshall, Le rôle de la langue 10). Pour éviter toute confusion avec le français créole louisianais, variété parlée aujourd'hui majoritairement par la population de couleur, dans cette étude nous utilisons le terme le français colonial.

${ }^{3}$ Etant donné que le recensement de 2000 (ainsi que celui de 1990) ne reflète pas précisément la situation linguistique de la population francophone de la Louisiane, en incluant le français cadien dans la catégorie de français, il est impossible d'identifier le nombre de locuteurs de cadien. Par ailleurs, les statistiques du même recensement montrent que 85414 Américains ont déclaré leur descendance acadienne/cadienne et 179739 Louisianais, la descendance canadienne, y compris acadienne. 